\title{
Biosynthesis of silver nanoparticles by the fungus Arthroderma fulvum and its antifungal activity against genera of Candida, Aspergillus and Fusarium
}

\author{
This article was published in the following Dove Press journal: \\ International Journal of Nanomedicine \\ 4 May 2016 \\ Number of times this article has been viewed
}

\section{Baiji Xue' \\ Dan $\mathrm{He}^{\prime}$ \\ Song Gao' \\ Dongyang Wang' \\ Koji Yokoyama ${ }^{2}$ \\ Li Wang'}

'Department of Pathogenobiology, Jilin University Mycology Research Center, Key Laboratory of

Pathobiology, Ministry of Education, College of Basic Medical Sciences, Jilin University, Changchun, People's Republic of China; ${ }^{2}$ Medical Mycology Research Center, Chiba University, Chiba, Japan
Correspondence: Li Wang

Department of Pathogenobiology, Jilin University Mycology Research Center, Key Laboratory of Pathobiology, Ministry of Education, College of Basic Medical Sciences, Jilin University, No 126

Xinmin Street, Changchun, Jilin I3002I,

People's Republic of China

Tel +8643I 856I 9486

Email wli620730@।26.com

\begin{abstract}
The objective of this study was to find one or more fungal strains that could be utilized to biosynthesize antifungal silver nanoparticles (AgNPs). Using morphological and molecular methods, Arthroderma fulvum was identified as the most effective fungal strain for synthesizing AgNPs. The UV-visible range showed a single peak at $420 \mathrm{~nm}$, which corresponded to the surface plasmon absorbance of AgNPs. X-ray diffraction and transmission electron microscopy demonstrated that the biosynthesized AgNPs were crystalline in nature with an average diameter of $15.5 \pm 2.5 \mathrm{~nm}$. Numerous factors could potentially affect the process of biosynthesis, and the main factors are discussed here. Optimization results showed that substrate concentration of $1.5 \mathrm{mM}$, alkaline $\mathrm{pH}$, reaction temperature of $55^{\circ} \mathrm{C}$, and reaction time of 10 hours were the optimum conditions for AgNP biosynthesis. Biosynthesized AgNPs showed considerable activity against the tested fungal strains, including Candida spp., Aspergillus spp., and Fusarium spp., especially Candida spp.
\end{abstract}

Keywords: silver nanoparticles, fungi, antifungal activity, nanomedicine

\section{Introduction}

Nanoparticles are particles of 1-100 nm diameter. They have unique properties such as a surface effect, an optical effect, a quantum size effect, and a macroscopic quantum tunneling effect. They also differ from conventional materials in how they exhibit heat, light, electricity, magnetism, catalysis, and sensitivity. ${ }^{1}$ Methods of synthesizing nanoparticles include chemical, physical, and biological protocols. Chemical and physical methods have traditionally been used to synthesize nanoparticles, but as "green" approaches increase in popularity, nanoparticles are increasingly being produced by nontoxic and environmentally friendly methods. The development of reliable and environmentally- friendly processes to synthesize nanoparticles is an important step in the application of nanotechnology. Biological methods have become increasingly prominent because they are inexpensive, use mild reaction conditions in a variety of hosts, and can produce stable nanoparticles of controlled dimensions. ${ }^{2-4}$ Biological methods include synthesis through the use of plants, ${ }^{5,6}$ bacteria, ${ }^{7,8}$ and fungi. ${ }^{9-12}$ Fungi have become one of the main biological candidates for synthesizing nanoparticles because of their metabolic diversity.

The first report of using fungi to biosynthesize nanoparticles dates back to a letter in Nature in 1989, reporting the production of CdSe nanoparticles by Candida albicans. ${ }^{13}$ 
Subsequent studies demonstrated that fungi could biosynthesize different metal nanoparticles, ${ }^{14-16}$ including AgNPs. The yeast strain MKY3 biosynthesizes AgNPs of 2-5 nm diameter extracellularly in its logarithmic growth phase. ${ }^{17}$ Verma et $\mathrm{al}^{18}$ and Qian et $\mathrm{l}^{19}$ have used endophytic fungi to biosynthesize AgNPs and have evaluated their antibacterial activity. Li et al ${ }^{20}$ utilized a soil isolate of Aspergillus terreus to biosynthesize AgNPs and discussed possible mechanisms of biosynthesis.

Interdisciplinary research and improved scientific knowledge have contributed to significant progress in the field of biology, especially with the emergence of nanotechnology, which has created a huge impact on all spheres of human life. AgNPs are important in medicine, particularly as "natural" antibacterial and antifungal agents in an era of drug resistance. AgNPs showed better antibacterial and antifungal activities against Pseudomonas aeruginosa, Escherichia coli, ${ }^{21}$ and Candida spp. ${ }^{22,23}$ Fungal infections are becoming more common; in addition to yeast being the main pathogenic bacteria, diseases caused by Aspergillus and Fusarium also continue to increase, ${ }^{24,25}$ and the limitations of drugs make it difficult to treat.

In this study, we first biosynthesized AgNPs using the fungus Arthroderma fulvum strain HT77 and then evaluated the antifungal properties of these AgNPs against an unprecedentedly broad diversity of fungal pathogens, including Candida spp., Aspergillus spp., and Fusarium spp. We have optimized the conditions of AgNP production in this system, and we discuss the effects of different conditions on the biosynthesis of AgNPs.

\section{Materials and methods Isolation of strain HT77}

Strain HT77 was one of 17 fungi isolated from soil samples collected from different sites of Nanhu Park, Jilin, People's Republic of China. Pure cultures were established by performing serial dilutions and plating onto potato dextrose agar (PDA; BD, Franklin Lakes, NJ, USA) medium. Plates were incubated at $28^{\circ} \mathrm{C}$ for 15 days and monitored daily to assess the growth of fungal colonies. Each purified fungal strain was checked by the hyphal tip method ${ }^{26}$ and transferred to a new PDA plate. Of the isolated strains, HT77 had the highest level of AgNP biosynthesis; it was stored at $4^{\circ} \mathrm{C}$ on PDA slopes for further study.

\section{Morphological observation and identification by ITS sequence analysis}

After storage, strain $\mathrm{HT} 77$ was reactivated on PDA at $28^{\circ} \mathrm{C}$ for 3 days and then inoculated onto a new PDA plate and grown at $28^{\circ} \mathrm{C}$ for 10 days. The macroscopic morphology was observed by eye. Cultures were incubated on slides of PDA at $28^{\circ} \mathrm{C}$ for 4 days; the slides were stained with lactophenol cotton blue and then observed under a light microscope (Nikon Alphaphot 2, YS2-H) to observe its micromorphology. ${ }^{19}$

Strain HT77 was inoculated into $10 \mathrm{~mL}$ of potato dextrose broth (BD) and incubated at $28^{\circ} \mathrm{C}$ for 3 days with continuous agitation at $140 \mathrm{rpm}$. Genomic DNA was extracted using the GenTLETM for Yeast kit (Takara Bio, Dalian, People's Republic of China) as described previously. ${ }^{27}$ Polymerase chain reaction amplification of the internal transcribed spacer region was performed as described previously (primers ITS1: 5'TCCGTAGGTGAACCTGCGG3'; ITS4: 5'TCCTCCGCTTATTGATATGC-3'). ${ }^{19}$ Polymerase chain reaction products were directly sequenced by Comate Bioscience (Jilin, People's Republic of China). Sequence editing and analysis were performed with ATGC and GENETYX software; homology studies were performed using BLASTn against the GenBank nr server. The ITS sequence of HT77 was submitted to GenBank.

\section{Biosynthesis of AgNPs}

Strain HT77 was grown in $100 \mathrm{~mL}$ cultures of potato dextrose broth, which had been inoculated with $\sim 10^{6}$ cells, in $250 \mathrm{~mL}$ conical flasks. The flasks were incubated at $28^{\circ} \mathrm{C}$ and $140 \mathrm{rpm}$ for 7 days. After incubation, fungal biomass was separated by filtration, washed with sterile distilled water to remove the traces of culture media components, resuspended in $100 \mathrm{~mL}$ distilled water, incubated at $28^{\circ} \mathrm{C}$ for 24 hours, and then filtered. Silver nitrate $\left(\mathrm{AgNO}_{3}, 1 \mathrm{mM}\right)$ was added to the filtrate to promote the formation of AgNPs. The ratio of cell filtrate to $\mathrm{AgNO}_{3}$ was kept at 1:9 (v/v), and the reaction mixture was incubated at $28^{\circ} \mathrm{C}$ for 48 hours. Controls (without the addition of $\mathrm{AgNO}_{3}$ ) were incubated under the same conditions.

\section{Analysis of AgNPs using UV-visible spectrophotometer}

Color change in the reaction mixture was the initial indicator of the formation of AgNPs. When the color changed, $3 \mathrm{~mL}$ of the reaction mixture was removed to measure its absorbance using a UV-visible spectrophotometer (UV-2450; Shimadzu, Tokyo, Japan), scanning the 300- to 600-nm absorbance spectrum in steps of $1 \mathrm{~nm}$. Scanning was performed after reaction times ranging from 5 minutes to 10 hours.

\section{Characterization of AgNPs by XRD and TEM}

The presence of AgNPs was confirmed by X-ray diffraction (XRD). Detailed characterization of the size, distribution, 
and morphology of AgNPs was performed using transmission electron microscopy (TEM). AgNPs were isolated by centrifugation of the reaction mixture at $10,000 \mathrm{rpm}$ for 10 minutes, washed twice with sterile distilled water, and then resuspended in $10 \mathrm{~mL}$ sterile distilled water. One drop of this resuspended sample was applied to a carbon-coated copper grid and allowed to dry before analysis. The average size of biosynthesized AgNPs was determined using a Malvern Zetasizer Nano ZS (Malvern Instruments, Malvern, UK) analyzer at room temperature.

\section{Optimization of AgNP biosynthesis}

Four main parameters were selected for the optimization of AgNP biosynthesis: substrate concentration, reaction $\mathrm{pH}$, reaction temperature, and reaction time. Each variable was optimized by varying only a single parameter at a time, that is, substrate concentration alone $(0.5 \mathrm{mM}, 1.0 \mathrm{mM}, 1.5 \mathrm{mM}$, $2.0 \mathrm{mM}$, and $2.5 \mathrm{mM} \mathrm{AgNO}_{3}$ ), reaction $\mathrm{pH}(5.0,6.0,7.0$, $8.0,9.0$, and 10.0$)$, reaction temperature $\left(15^{\circ} \mathrm{C}, 25^{\circ} \mathrm{C}, 35^{\circ} \mathrm{C}\right.$, $45^{\circ} \mathrm{C}, 55^{\circ} \mathrm{C}$, and $65^{\circ} \mathrm{C}$ ), and reaction time ( 2 hours, 4 hours, 6 hours, 8 hours, 10 hours, and 12 hours). The absorbance of resultant samples was measured at $420 \mathrm{~nm}$.

\section{Analysis of the antifungal activity of AgNPs}

Ten species from three genera were tested for their susceptibility to the antifungal properties of biosynthesized AgNPs, including C. albicans ATCC90028, C.parapsilosis ATCC22019, C. krusei ATCC6258, C. tropicalis JLCC31384, Aspergillus fumigatus IFM40808, A. flavus IFM55648, A. terrrus JLCC30844, Fusarium solani JLCC30866, $F$. moniliforme JLCC31463, and F. oxysporum JLCC31768 (ATCC: American Type Culture Collection, Manassas, VA, USA; IFM: Institute for Food Microbiology, at present the Medical Mycology Research Center, Chiba University, Japan; JLCC: Culture Collection of Jilin University, Mycology Research Center, People's Republic of China).

Using protocols based on the Clinical and Laboratory Standards Institute guidelines (document M38-A2), the minimal inhibitory concentrations (MICs) of the AgNPs were determined for the tested fungi, using a broth microdilution method. AgNPs were added to sterile RPMI-1640 medium containing $2 \%(\mathrm{w} / \mathrm{v})$ glucose, buffered to $\mathrm{pH}$ 7.0. Final concentrations of AgNPs were $0.125-64 \mathrm{mg} / \mathrm{L}$. The final inoculation concentration in cultures of the tested strains was 1.0-2.0 $\times 10^{4} \mathrm{CFU} / \mathrm{mL}$. Itraconazole and fluconazole were used as positive controls. Testing was performed in 96-well plates, which were incubated at $37^{\circ} \mathrm{C}$ for yeasts or $28^{\circ} \mathrm{C}$ for filamentous fungi. The plates were assessed visually after 24 hours for Candida spp. and after 48 hours for Aspergillus spp. and Fusarium spp. The MIC was defined as the lowest concentration of the AgNPs that inhibited visible fungal growth by $80 \%$. Tests were repeated three times.

\section{Fungal growth kinetics in the presence of AgNPs}

The effect of AgNPs on the kinetics of fungal growth was studied for Candida spp. (C. albicans ATCC90028, C. parapsilosis ATCC22019, C. krusei ATCC6258, and C. tropicalis JLCC31384). Briefly, yeast extract peptone dextrose medium (YEPD; BD) containing $2.50 \mu \mathrm{g} / \mathrm{mL}$ AgNPs was inoculated with fungal cells to achieve an initial cell density of $1 \times 10^{6} \mathrm{CFU} / \mathrm{mL}$. Cultures were incubated in $150 \mathrm{~mL}$ conical flasks containing $30 \mathrm{~mL}$ of YEPD supplemented with AgNPs and shaken at $37^{\circ} \mathrm{C}$ and $100 \mathrm{rpm}$ for 36 hours. Growth curves were drawn using the optical density $\left(\mathrm{OD}_{560}\right)$ values measured during growth.

\section{Results and discussion}

\section{Strain identification}

The HT77 strain that could rapidly biosynthesize AgNPs, was obtained through the experiment. This strain grew slowly; the colonies incubated on PDA medium at $28^{\circ} \mathrm{C}$ were $6.5 \mathrm{~cm}$ in diameter at day 9. Each colony was suede-like and released red pigment into the PDA (Figure 1A). Conidia were globose to pyriform with $15-25 \mu \mathrm{m}$ in length, darkly pigmented, had a verrucose external surface, and became multicellular (Figure 1B). Based on these typical characteristics, the strain was identified as the genus Arthroderma. BLAST analysis of the ITS sequence of fungus returned 99\% homology to the ITS sequence from Arthroderma fulvum (accession number AB193716.1). Based on the degree of homology typically seen in ITS sequence-related fungi, strain HT77 was identified as Arthroderma fulvum finally.

\section{Biosynthesis and characterization of AgNPs}

When $\mathrm{AgNO}_{3}$ was added to the fungal cell filtrate, the color of the reaction mixture turned dark brown after 1 hour (Figure 2), indicating surface plasmon resonance of metallic AgNPs. The formation of AgNPs was detected and monitored over time by UV-visible absorption spectrum scanning in the range of 300-600 $\mathrm{nm}$ (Figure 3), with increasing reaction time (ranging from 5 minutes to 10 hours). As illustrated in the UV-visible spectra, the absorbance intensity gradually increased with time without any shift of the wavelength 

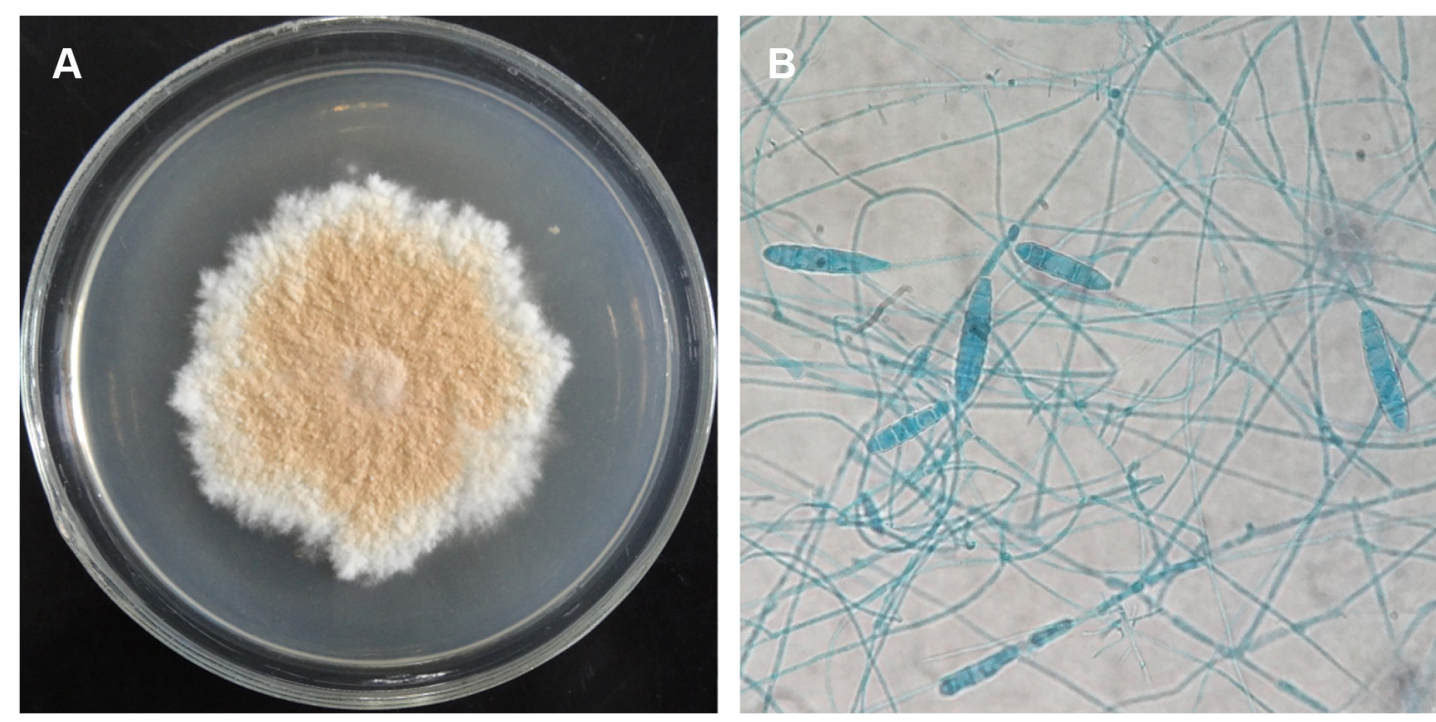

Figure I Morphology of Arthroderma fulvum (strain HT77).

Notes: (A) Macroscopic morphology (PDA, $28^{\circ} \mathrm{C}, 9$ days) and (B) microscopic morphology $(\times 400$, stained with lactophenol cotton blue).

Abbreviation: PDA, potato dextrose agar.

in which the maximum absorbance was observed. This indicated a continuous reduction of $\mathrm{AgNO}_{3}$ and, consequently, an increase in AgNPs concentration.

Figure 3 shows that the reaction had run for 5 minutes; a specific absorption peak could be detected at $420 \mathrm{~nm}$, indicating the formation of AgNPs. The formation time of AgNPs is shorter compared with some previous studies..$^{28,29}$ After the reaction had run for 10 hours, the specific surface plasmon resonance band was at $421 \mathrm{~nm}$, remaining close to $420 \mathrm{~nm}$. Absorption values had reached their maximum,

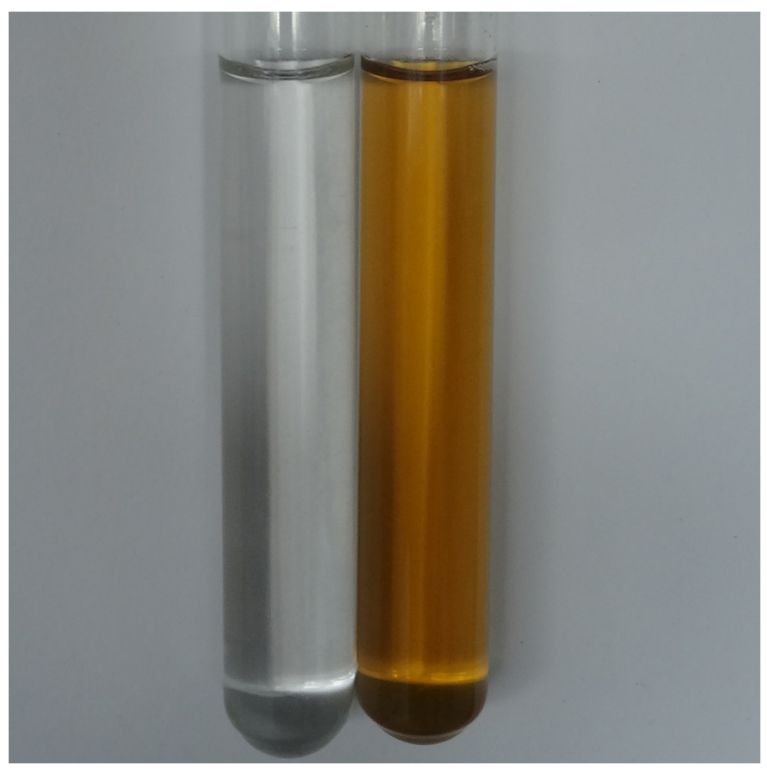

Figure 2 The cell filtrate of Arthroderma fulvum without $\mathrm{AgNO}_{3}$ (left) and with $\mathrm{AgNO}_{3}$ after I hour (right). demonstrating that AgNPs were both dispersed and stable throughout the reaction volume. Arthroderma fulvum has never previously been reported to biosynthesize AgNPs, making our work an important step toward green methods of nanoparticle synthesis.

XRD was used to identify the crystalline nature of AgNPs. The XRD patterns of the lyophilized AgNPs using Arthroderma fulvum are shown in Figure 4. Five diffraction peaks at $2 \theta$ values of $31.8^{\circ}, 37.9^{\circ}, 45.0^{\circ}, 64.3^{\circ}$, and $77.7^{\circ}$ were observed, corresponding to Bragg's reflections of metallic AgNPs crystallized in a face-centered cubic structure with basal (111), (200), (220), (311), and (222) lattice planes, respectively. ${ }^{19,20}$ The results of the XRD pattern further corroborated the biosynthesis of AgNPs, with

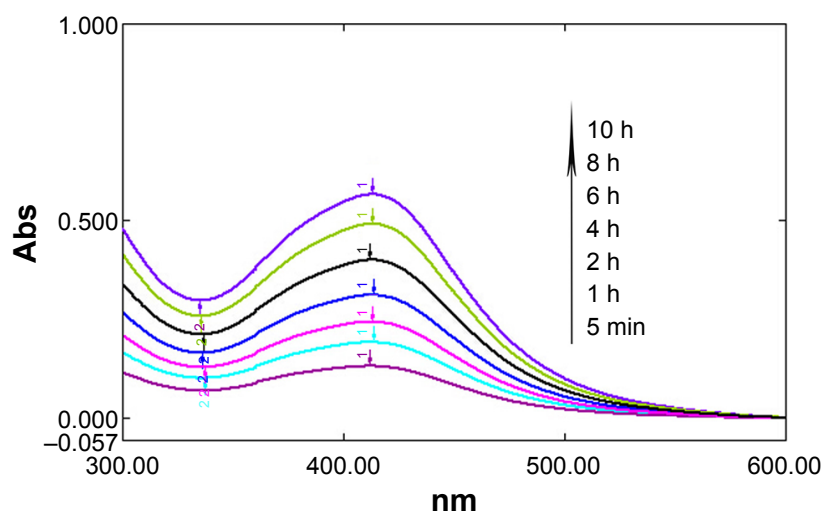

Figure $3 \mathrm{UV}$-visible absorption spectrum of AgNPs biosynthesized by the reduction of $\mathrm{AgNO}_{3}$ solution with the cell filtrate of Arthroderma fulvum with different time intervals.

Abbreviations: Abs, absorbance; AgNPs, silver nanoparticles; h, hours; min, minutes. 


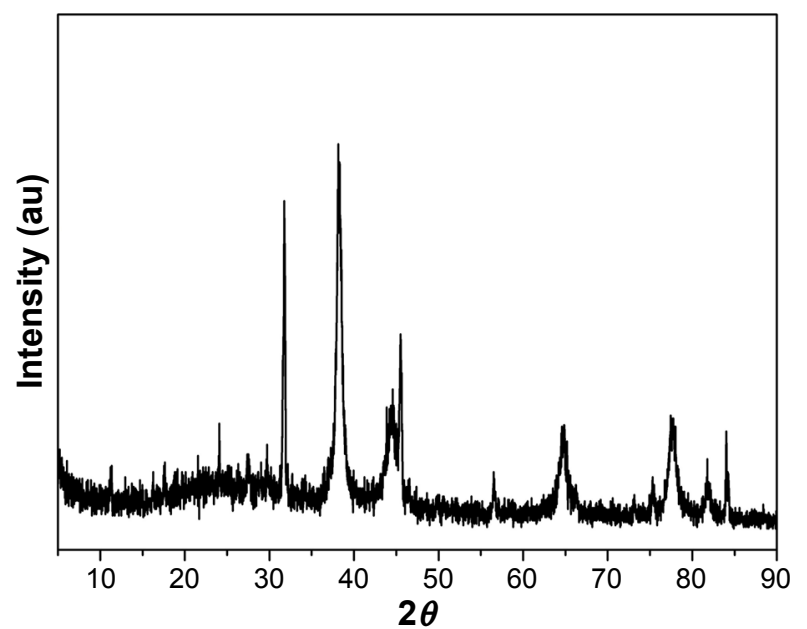

Figure 4 XRD patterns of lyophilized AgNPs biosynthesized by the reduction of $\mathrm{AgNO}_{3}$ solution with the cell filtrate of Arthroderma fulvum.

Abbreviations: XRD, X-ray diffraction; AgNPs, silver nanoparticles.

sharp bands of Bragg peaks corresponding to crystalline AgNPs, which may result from stabilization of the AgNPs by reducing agents in the reaction. The present results clearly illustrated that AgNPs biosynthesized by this green method are nanocrystalline in nature. ${ }^{30}$

A TEM image of the AgNPs is shown in Figure 5A, showing that they are spherical in shape. The average diameter was $15.5 \pm 2.5 \mathrm{~nm}$, with a highly uniform and narrow distribution of diameters (data not shown). Similar observations have previously been reported by Jaidev and Narasimha. ${ }^{31}$ A Malvern Zetasizer Nano ZS was used to analyze the diameters more precisely. Figure $5 \mathrm{~B}$ shows an average diameter of $20.56 \mathrm{~nm}$, with $5.8 \%$ of the particles being $<1.92 \mathrm{~nm}$ and $94.2 \%$ being $<36.10 \mathrm{~nm}$ in diameter, which was similar to the TEM data. The AgNPs were monodispersed with a low polydispersity index of 0.27 . The size measured by TEM micrographs was slightly smaller than that measured by Malvern Zetasizer Nano ZS. This may have been caused by the presence of attached surface proteins, carbohydrates, and other cellular materials, which would have been measured by the Zetasizer but would not have been retained in a vacuum under an electron beam. This may also explain the reason for the stable presence of biosynthesized nanoparticles.

In order to further illustrate the stability, the size analysis of AgNPs (synthesized for 2 months) was carried out again. Figure 5C shows an average diameter of $20.77 \mathrm{~nm}$, with $5.7 \%$ of the particles being $<1.97 \mathrm{~nm}$ and $94.2 \%$ being $<36.15 \mathrm{~nm}$ in diameter, which was basically the same with Figure 5B. It can be seen that, after 2 months of the placement, the changes of the distribution and size were
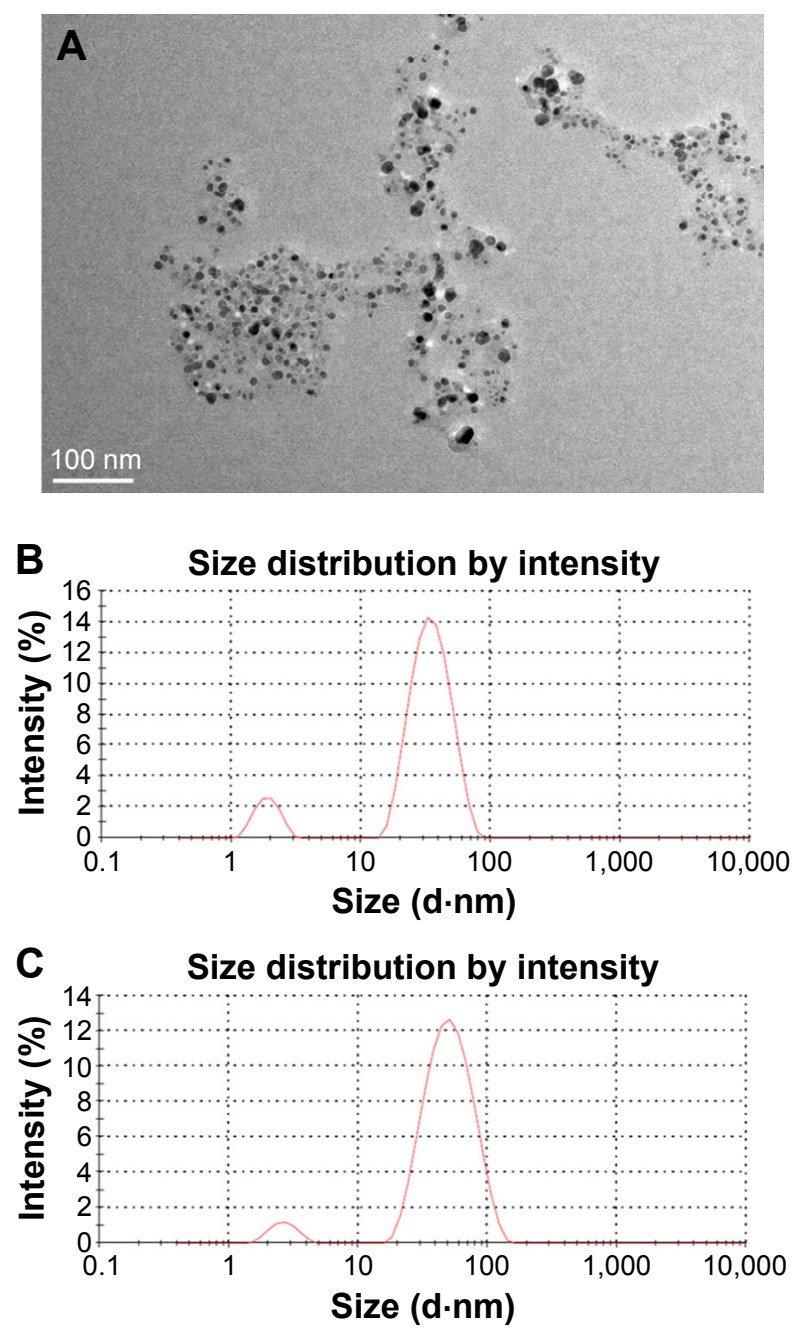

Figure 5 TEM image and size distribution of the AgNPs.

Notes: (A) Representative images of AgNPs biosynthesized by the reduction of $\mathrm{AgNO}_{3}$ solution with the cell filtrate of Arthroderma fulvum (scale bar $=100 \mathrm{~nm}$ ) and (B, C) size distribution of the AgNPs from Malvern Zetasizer Nano ZS analysis after being biosynthesized 10 hours and 2 months, respectively.

Abbreviations: AgNPs, silver nanoparticles; TEM, transmission electron microscopy.

small, indicating that the biosynthesized AgNPs showed appropriate stability.

The results from UV-visible absorption spectroscopy, XRD, and TEM all demonstrate that AgNPs were biosynthesized from extracellular filtrate. Therefore, we used a simple process to complete biosynthesis of AgNPs, which required very little complex equipment compared with chemical and physical methods. Arthroderma fulvum represents a promising candidate for large-scale production of AgNPs.

\section{Optimization of reaction conditions of biosynthesis of AgNPs}

Figure 6A shows that different substrate concentrations of $\mathrm{AgNO}_{3}$ in the reaction mixture had an obvious influence on the biosynthesis of AgNPs, and $1.5 \mathrm{mM}$ was the optimum 
concentration. When the substrate concentration reached $5 \mathrm{mM}$, the biosynthesis of AgNPs was both reduced and unstable, with aggregates and precipitation present at the bottom of the reaction container (data not shown). Optimal $\mathrm{pH}$ conditions for AgNP biosynthesis have varied by microbial
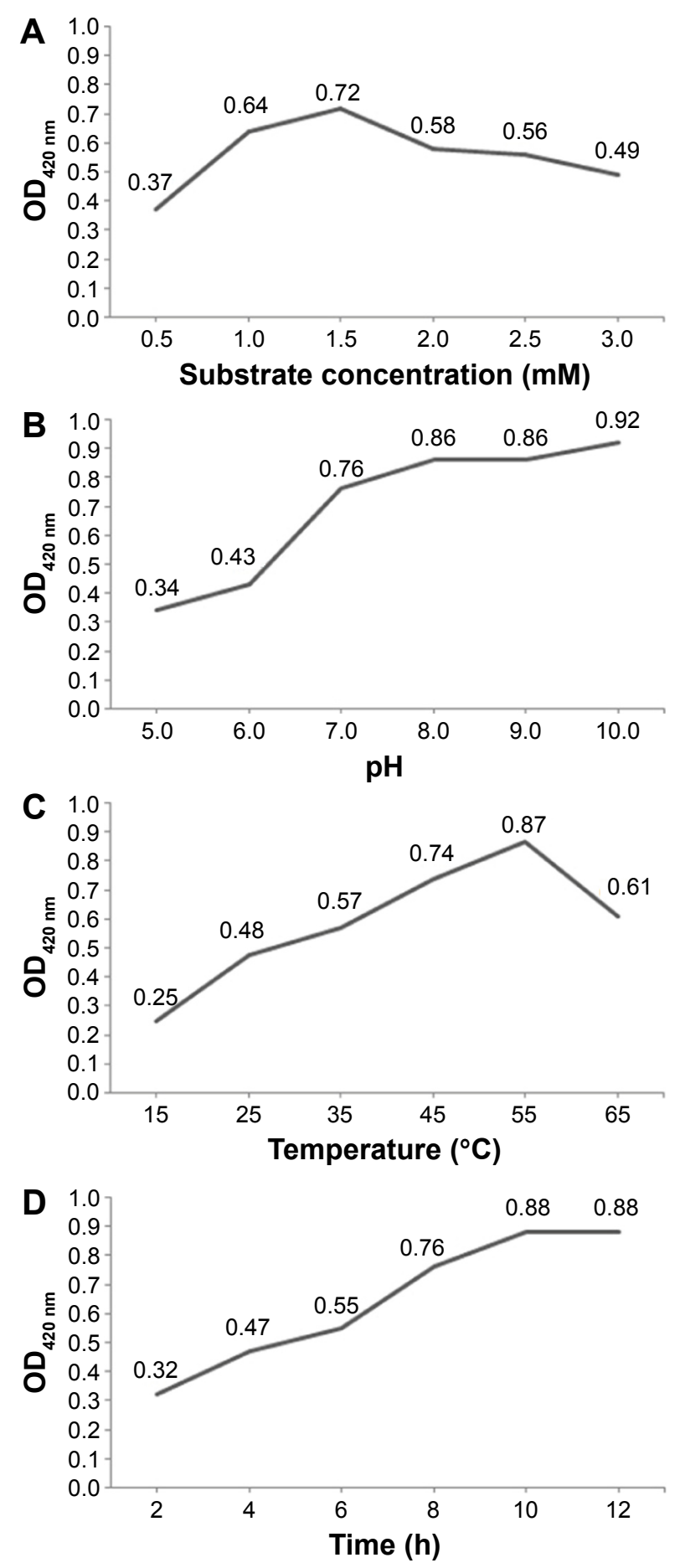

Figure 6 Optimization of reaction conditions of biosynthesis of AgNPs. Notes: Effect of $(\mathbf{A})$ substrate concentration, $(\mathbf{B}) \mathrm{pH},(\mathbf{C})$ temperature, and (D) time on $\mathrm{AgNP}$ biosynthesis by the reduction of $\mathrm{AgNO}_{3}$, solution with the cell filtrate of Arthroderma fulvum.

Abbreviations: AgNPs, silver nanoparticles; OD, optical density; h, hours. strain in the published literature. ${ }^{32}$ Here, a $\mathrm{pH}$ range of 5-10 was selected for the study. Absorbance increased with $\mathrm{pH}$, suggesting that an alkaline environment was more suitable for AgNP biosynthesis (Figure 6B). The optimal temperature for AgNP biosynthesis was $55^{\circ} \mathrm{C}$, with production reducing at higher temperatures such as $65^{\circ} \mathrm{C}$, as demonstrated by absorbance measurements (Figure 6C). Absorbance values also increased with time, up to 10 hours, but did not increase thereafter (Figure 6D).

The results of our optimization experiments were similar to those of Qian et al, ${ }^{19}$ suggesting that yield and stability of AgNP biosynthesis can be affected by several different parameters.

\section{Analysis of the antifungal activity of AgNPs}

Because of the limitations of antifungal drugs, fungal infections are increasingly widespread. Here, we investigated the antifungal activity of AgNPs on ten fungal pathogens, including Candida spp., Aspergillus spp., and Fusarium spp. MIC values for the tested agents are presented in Table 1. AgNPs, with an MIC in the range of $0.125-4.00 \mu \mathrm{g} / \mathrm{mL}$, showed significant antifungal activity against all tested fungi. Fluconazole, with an MIC in the range of $0.250-16.00 \mu \mathrm{g} / \mathrm{mL}$, only exhibited an antifungal effect against Candida spp. Itraconazole, with an MIC in the range of $0.030-0.250 \mu \mathrm{g} / \mathrm{mL}$, displayed strong antifungal activity against all tested species except Fusarium spp. The present results demonstrate that the AgNPs exhibited high antifungal activity, in accordance with several previous reports. ${ }^{33-35}$ The MIC values obtained here were similar to those reported by Qian et al. ${ }^{19}$ At concentrations around $1 \mathrm{mg} / \mathrm{mL}, \mathrm{AgNPs}$ displayed a broader antifungal spectrum than itraconazole and fluconazole, the common antifungal agents. These results strongly suggested that the biosynthesized AgNPs could be a potent antifungal agent.

Table I Antifungal activity of AgNPs, fluconazole, and itraconazole

\begin{tabular}{llll}
\hline \multirow{2}{*}{$\begin{array}{l}\text { Tested fungal } \\
\text { strains }\end{array}$} & \multicolumn{3}{l}{ MIC $(\mu \mathrm{g} / \mathrm{mL})$} \\
\cline { 2 - 4 } & AgNPs & Fluconazole & Itraconazole \\
\hline Candida albicans & 0.500 & 0.250 & 0.030 \\
Candida parapsilosis & 0.125 & 8.00 & 0.250 \\
Candida krusei & 0.125 & 16.0 & 0.250 \\
Candida tropicalis & 0.250 & 0.250 & 0.250 \\
Aspergillus fumigatus & 1.00 & $>64.0$ & 0.030 \\
Aspergillus flavus & 2.00 & $>64.0$ & 0.125 \\
Aspergillus terrus & 1.00 & $>64.0$ & 0.250 \\
Fusarium solani & 2.00 & $>64.0$ & $>16.0$ \\
Fusarium moniliforme & 4.00 & $>64.0$ & $>16.0$ \\
Fusarium oxysporum & 2.00 & $>64.0$ & $>16.0$ \\
\hline
\end{tabular}

Abbreviations: AgNPs, silver nanoparticles; MIC, minimal inhibitory concentration. 
Yeast is the main cause of fungal disease, which means that there is considerable motivation for finding antifungal agents against yeasts. In this study, we studied the effect of AgNPs on the growth of yeast by adding AgNPs into YEPD. The growth curves of Candida (C.albicans, C.parapsilosis, C. krusei, and C. tropicalis) in the presence of AgNPs are shown in Figure 7, indicating that growth can be completely inhibited by AgNPs, that is, they are fungistatic, as also shown in Table 1. The inhibition of $C$. parapsilosis and $C$. krusei was stronger than that of C. tropicalis and C. albicans at similar concentrations.

Although infections caused by filamentous fungi Aspergillus and Fusarium are increasingly being reported, most studies have concentrated on yeasts belonging to Candida spp. ${ }^{19,20,22,23}$ In our research, we also studied the effects of AgNPs on members of Aspergillus and Fusarium. Thus, our results should provide a valuable reference for the future treatment of fungal diseases. Notwithstanding, further studies should be undertaken to understand the toxicity of AgNPs before developing them for clinical applications.

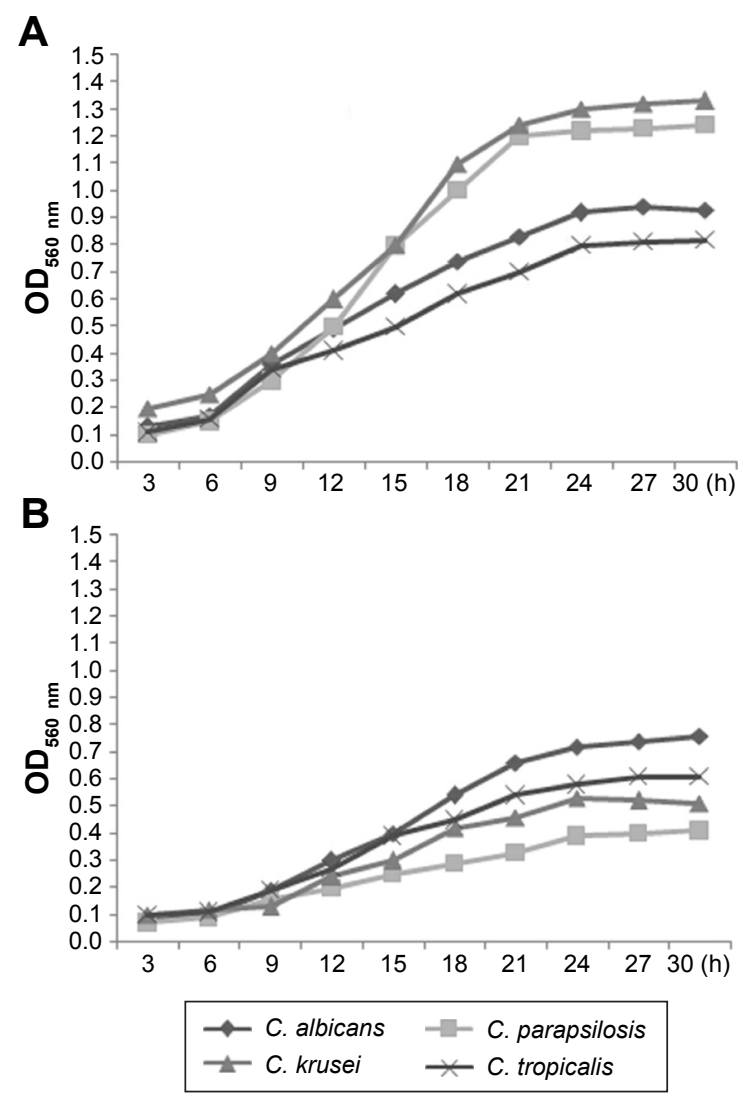

Figure 7 Fungal growth kinetics in the presence of AgNPs.

Notes: The growth curves of Candida (C. albicans, C. parapsilosis, C. krusei, and C. tropicalis) in the absence of $\operatorname{AgNPs}(\mathbf{A})$ and the presence of $\mathrm{AgNPs}(\mathbf{B})$.

Abbreviations: C. albicans, Candida albicans; C. parapsilosis, Candida parapsilosis; C. krusei, Candida krusei; C. tropicalis, Candida tropicalis; AgNPs, silver nanoparticles; OD, optical density; h, hours.

\section{Conclusion}

This study demonstrated that a soil-isolated strain of Arthroderma fulvum could perform biosynthesis of AgNPs. The biosynthesized AgNPs showed appropriate uniformity and stability and showed considerable antifungal activity against Candida spp., Aspergillus spp., and Fusarium spp. Optimization of AgNP biosynthesis was performed and will permit the large-scale production of AgNPs. The ability to biosynthesize AgNPs using Arthroderma fulvum is highly promising as a green, sustainable, simple, and easily disseminated method of producing metal nanoparticles.

\section{Acknowledgment}

This work was funded by the National Natural Science Foundation of major international cooperation projects of the People's Republic of China (No 30910103903).

\section{Disclosure}

The authors report no conflicts of interest in this work.

\section{References}

1. Sau TK, Rogach AL, Jäckel F, Klar TA, Feldmann J. Properties and applications of colloidal nonspherical noble metal nanoparticles. Adv Mater. 2010;22(16):1805-1825.

2. Ajitha B, Ashok Kumar Reddy Y, Sreedhara Reddy P. Green synthesis and characterization of silver nanoparticles using Lantana camara leaf extract. Mater Sci Eng C Mater Biol Appl. 2015;49:373-381.

3. Kuppusamy P, Ichwan SJ, Parine NR, Yusoff MM, Maniam GP, Govindan N. Intracellular biosynthesis of $\mathrm{Au}$ and $\mathrm{Ag}$ nanoparticles using ethanolic extract of Brassica oleracea L. and studies on their physicochemical and biological properties. J Environ Sci (China). 2015; 29:151-157.

4. Guo M, Li W, Yang F, Liu H. Controllable biosynthesis of gold nanoparticles from a Eucommia ulmoides bark aqueous extract. Spectrochim Acta A Mol Biomol Spectrosc. 2015;142:73-79.

5. Ajitha B, Reddy YA, Reddy PS. Biosynthesis of silver nanoparticles using Momordica charantia leaf broth: Evaluation of their innate antimicrobial and catalytic activities. J Photochem Photobiol B Biol. 2015;146:1-9.

6. Latha M, Sumathi M, Manikandan R, Arumugam A, Prabhu NM Biocatalytic and antibacterial visualization of green synthesized silver nanoparticles using Hemidesmus indicus. Microb Pathog. 2015;82: 43-49.

7. Sohm B, Immel F, Bauda P, Pagnout C. Insight into the primary mode of action of $\mathrm{TiO}_{2}$ nanoparticles on Escherichia coli in the dark. Proteomics. 2015;15(1):98-113.

8. Srivastava P, Braganca JM, Kowshik M. In vivo synthesis of selenium nanoparticles by Halococcus salifodinae BK18 and their antiproliferative properties against HeLa cell line. Biotechnol Prog. 2014; 30(6):1480-1487.

9. Salvadori MR, Nascimento CA, Corrêa B. Nickel oxide nanoparticles film produced by dead biomass of filamentous fungus. Sci Rep. 2014; 4:6404.

10. Tidke PR, Gupta I, Gade AK, Rai M. Fungus-mediated synthesis of gold nanoparticles and standardization of parameters for its biosynthesis. IEEE Trans Nanobioscience. 2014;13(4):397-402.

11. Soni N, Prakash S. Fungal-mediated nano silver: an effective adulticide against mosquito. Parasitol Res. 2012;111(5):2091-2098. 
12. Zikmundová M, Drandarov K, Bigler L, Hesse M, Werner C. Biotransformation of 2-benzoxazolinone and 2-hydroxy-1,4-benzoxazin-3-one by endophytic fungi isolated from Aphelandra tetragona. Appl Environ Microbiol. 2002;68(10):4863-4870.

13. Dameron CT, Reeser RN, Mehra RK, et al. Biosynthesis of cadmium sulfide quantum semiconductor nanocrystallites. Nature. 1989;338 (6216): 596-597.

14. Jha AK, Prasad K, Kulkarni AR. Synthesis of TiO2 nanoparticles using microorganisms. Colloids Surf B Biointerfaces. 2009;71(2):226-229.

15. Zare B, Babaie S, Setayesh N, Shahverdi AR. Isolation and characterization of a fungus for extracellular synthesis of small selenium nanoparticles. Nanomed J. 2013;1(1):13-19.

16. Jha AK, Prasad K, Prasad K. A green low-cost biosynthesis of $\mathrm{Sb}_{2} \mathrm{O}_{3}$ nanoparticles. Biochem Eng J. 2009;43:303-306.

17. Kowshik M, Ashtaputre S, Kharrazi S, et al. Extracellular synthesis of silver nanoparticles by a silver-tolerant yeast strain MKY3. Nanotechnology. 2003;14:95-100.

18. Verma VC, Kharwar RN, Gange AC. Biosynthesis of antimicrobial silver nanoparticles by the endophytic fungus Aspergillus clavatus. Nanomedicine (Lond). 2010;5(1):33-40.

19. Qian Y, Yu H, He D, et al. Biosynthesis of silver nanoparticles by the endophytic fungus Epicoccum nigrum and their activity against pathogenic fungi. Bioprocess Biosyst Eng. 2013;36(11):1613-1619.

20. Li G, He D, Qian Y, et al. Fungus-mediated green synthesis of silver nanoparticles using Aspergillus terreus. Int J Mol Sci. 2012; 13(1):466-476.

21. Mohan S, Oluwafemi OS, George SC, et al. Completely green synthesis of dextrose reduced silver nanoparticles, its antimicrobial and sensing properties. Carbohydr Polym. 2014;106:469-474.

22. Panácek A, Kolár M, Vecerová R, et al. Antifungal activity of silver nanoparticles against Candida spp. Biomaterials. 2009;30(31): 6333-6340.

23. Rahisuddin, Al-Thabaiti SA, Khan Z, Manzoor N. Biosynthesis of silver nanoparticles and its antibacterial and antifungal activities towards Gram-positive, Gram-negative bacterial strains and different species of Candida fungus. Bioprocess Biosyst Eng. 2015;38(9):1773-1781.

24. Gomes CC, Pinto LC, Victor FL, et al. Aspergillus in endodontic infection near the maxillary sinus. Braz J Otorhinolaryngol. 2015;81(5):527-532.
25. Garcia RR, Min Z, Narasimhan S, Bhanot N. Fusarium brain abscess: case report literature review. Mycoses. 2015;58(1):22-26.

26. Strobel G, Yang X, Sears J, Kramer R, Sidhu RS, Hess WM. Taxol from Pestalotiopsis microspora, an endophytic fungus of Taxus wallachiana. Microbiology. 1996;142(Pt 2):435-440.

27. Yokoyama K, Biswas SK, Miyaji M, Nishimura K. Identification and phylogenetic relationship of the most common pathogenic Candida species inferred from mitochondrial cytochrome $\mathrm{b}$ gene sequences. J Clin Microbiol. 2000;38(12):4503-4510.

28. Mukherjee P, Ahmad A, Mandal D, et al. Fungus-mediated synthesis of silver nanoparticles and their immobilization in the mycelial matrix: a novel biological approach to nanoparticle synthesis. Nano Lett. 2001; 1(10):515-519.

29. Sadowski Z, Maliszewska IH, Grochowalska B, Polowczyk I, Kozlecki T. Synthesis of silver nanoparticles using microorganisms. Mater Sci Poland. 2008;26:419-424.

30. Nabikhan A, Kandasamy K, Raj A, Alikunhi NM. Synthesis of antimicrobial silver nanoparticles by callus leaf extracts from saltmarsh plant Sesuvium portulacastrum L. Colloids Surf B Biointerfaces. 2010; 79(2):488-493.

31. Jaidev LR, Narasimha G. Fungal mediated biosynthesis of silver nanoparticles, characterization and antimicrobial activity. Colloids Surf B Biointerfaces. 2010;81(2):430-433.

32. Kathiresan K, Manivannan S, Nabeel MA, Dhivya B. Studies on silver nanoparticles synthesized by a marine fungus, Penicillium fellutanum isolated from coastal mangrove sediment. Colloids Surf B Biointerfaces. 2009;71(1):133-137.

33. Petica A, Gavriliu S, Lungu M, Buruntea N, Panzaru C. Colloidal silver solutions with antimicrobial properties. Mat Sci Eng B Adv. 2008;152(1-3): $22-27$.

34. Kim KJ, Sung WS, Moon SK, Choi JS, Kim JG, Lee DG. Antifungal effect of silver nanoparticles on dermatophytes. J Microbiol Biotechnol. 2008;18(8):1482-1484.

35. Gajbhiye M, Kesharwani J, Ingle A, Gade A, Rai M. Fungus-mediated synthesis of silver nanoparticles and their activity against pathogenic fungi in combination with fluconazole. Nanomedicine. 2009;5(4):382-386.
International Journal of Nanomedicine

\section{Publish your work in this journal}

The International Journal of Nanomedicine is an international, peerreviewed journal focusing on the application of nanotechnology in diagnostics, therapeutics, and drug delivery systems throughout the biomedical field. This journal is indexed on PubMed Central, MedLine, CAS, SciSearch $®$, Current Contents ${ }^{\circledR} /$ Clinical Medicine,

\section{Dovepress}

Journal Citation Reports/Science Edition, EMBase, Scopus and the Elsevier Bibliographic databases. The manuscript management system is completely online and includes a very quick and fair peer-review system, which is all easy to use. Visit http://www.dovepress.com/ testimonials.php to read real quotes from published authors. 